\title{
The Function of Physical Education in Promoting the Physical and Mental Health of College Students
}

\author{
Pengfei Niu ${ }^{1, *}$ Biqu Erga ${ }^{1}$
}

\begin{abstract}
${ }^{1}$ School of Physical Education, Sichuan Agricultural University, Ya'an, Sichuan 625014, China *Corresponding author.
\end{abstract}

\begin{abstract}
By analyzing the current social reality and development trend, this paper gives an analysis of the latent function and obvious function from the perspective of structural function theory, and concludes that if we only emphasize scores and achievements and despise the development of students' physical and mental balance, it will lead to the phenomenon of yin-yang imbalance in talent education and talent training. In order to correct this extreme behavior as soon as possible, we should aim at health and take physical exercise as the starting point to promote the all-round development of students.
\end{abstract}

Keywords: sports, education, function

\section{INTRODUCTION}

In the era of scientific and technological rationality, with the further strengthening of competition in various fields, adolescent health, especially the physical and mental health of college students, has gradually become a companion problem in the information and electronic age. To this end, the government, society and various major disciplines are involved in the field of physical and mental health of college students with different knowledge. Sports, as a way of physical exercise can regulate the special role of mind and psychology, and has all aspects of the function.

\section{QUESTION RAISED}

The World Health Organization estimates that global child mental disorders will increase by 50 per cent by 2020, making them the leading cause of disease, death and disability. The mental health status of children and adolescents in our country is also not optimistic, and it is estimated that at least 30 million of the children and adolescents under the age of 17 are suffering from various psychological problems.

$\mathrm{Wu}$ Guanghong conducted a survey on the mental health status of college students in the eastern, western and central regions, and found that students with moderate or higher mental health problems accounted for about $16 \%$ of the population of college students in China compared with the normal population in China. In recent years, problems caused by college students' physical and mental health have occurred frequently. Almost every university, middle school, even primary school has physical and mental health problems, and there is a trend of younger age. Faced with this problem, various disciplines are studying countermeasures and schemes. According to the suggestion of China Health and Health Commission, the state should attach great importance to this phenomenon, and start from the aspect of "treating disease without disease", focusing on prevention, and paying full attention to the cultivation and education of college students. The authors will analyze the effects of physical education on college students' physical and mental health from the perspective of functionalism.

\section{FUNCTIONAL THEORIES}

Functionalism is an important school for analyzing the law of social operation in western sociological circles. The theory of functionalism mainly focuses on the results brought after social operation, social behavior and so on, mostly staying in the four dimensions of function, such as positive function, negative function, latent function and obvious function.

This school began with the representative of functionalism of classical sociology, turgan, has not stopped the refinement of functionalism, dig deep and explore, and even later the school in Britain, Europe, Germany and Frankfurt have a very important influence, later functionalism with the social changes, the development of disciplines, functionalism with the movement of sociology to North America, eventually developed into a mature and perfect school. In the United States, sociologists, represented by Parsons and Robert K. Merton, have done more elaborate research on functionalism. Therefore, functionalism has the structural function of paying attention to macroscopic and preferring static society doctrine, through the functions of the four action systems, such as social adaptation, goal-attainment, cultural integration, and potential pattern maintenance, namely, the AGIL 
functional model. Although structural functionalism is beneficial to explain the operation of society, it is questioned by academic circles because the theoretical structure and system pay too much attention to and explain the objects. Therefore, Morton developed the theory suitable for mesoscopic on the basis of structural functionalism, known as mesoscopic theory and so on, among which, latent function, obvious function has the most extensive influence. Its explicit function refers to the function and function of each component in a particular social structure, one part can know its obvious function by observable and testable way, while the other part is called latent function which is not necessarily obtained by observing and verifying the immediate result or function, but only after a period of development change. The explicit function is helpful to further explore the latent function, which in turn can supplement the explicit function, which plays an important role in the movement, development and change of things. The author mainly discusses the function of physical education to college students' physical and mental health with the help of Morton's two functional theories. On this basis, it is suggested that the university education should pay attention to the simultaneous development of body and mind, in order to promote the all-round and sufficient education of college students, and to train the talents with all-round development of morality, intelligence and body for the country.

\section{THE FUNCTION OF PHYSICAL EDUCATION TO COLLEGE STUDENTS' PHYSICAL AND MENTAL HEALTH}

\section{A. The remarkable function of physical education on the physical and mental development of college students}

Physical education is to pay attention to the health of education activities, conducive to the health of students' physique. In ancient Greece, sports included games, wrestling, gymnastics and other related education content. As early as the 17th-18th century, western education was only something similar to horse riding, fencing, swimming, hunting, running, jumping and so on, especially sports activities, rather than what is closely related to education embodied in today's pedagogy. From the end of the 18th century to the beginning of the 19th century, the series of activities related to physical competition were widely spread in Europe and the United States, and gradually developed into today's pedagogy refers to "sports ", especially" physical activity as a means of education "this whole new concept. Modern sports are divided into two meanings: broad sense and narrow sense. Sports in a narrow sense refer to physical education. The concept here holds that "sports is a social and cultural phenomenon or educational process with physical activity as the medium, seeking individual physical and mental health and all-round development as the direct purpose, and taking the cultivation of perfect social citizens as the ultimate goal". This definition of sports not only explains its essential attributes, but also points out its category of belonging, and also distinguishes itself from the social phenomenon of its proximity or similarity. When applied in a broad sense, it generally refers to sports, which includes three aspects: physical education, competitive sports and physical exercise.

With the rise and popularization of the sports subject, its multiple functions will soon be effective. Sports have its own fitness, health heart of the two major characteristics. First of all, it is the internal and external integration of fitness characteristics. The object of physical education teaching is students, and the effect of physical education teaching should be reflected in the students. Students have strong plasticity, every conception and step of physical education teaching will directly affect the growth of students. The effect of a right teaching on students is not only the strength of the external muscle and the smoothness of the muscle lines, the perfect development of the bones, the sound of the visceral organs, but also the symmetry of the whole, the coordinated development, and the orderly and comprehensive development according to the sequence of growth and development. Physical education and internal and external integration of the fitness system reflects the order of physical development and comprehensiveness. Secondly, the fitness characteristics of the unity of body and mind: education focuses on the combination of physical beauty from the inside out and intellectual beauty from the outside. If the relationship between the two is separated, it is not conducive to the overall development of human beings. The wisdom of Chinese philosophy emphasizes the complementarity and overall unity of things. The Book of Changes, as the essence of Chinese traditional culture, is called the head of the Six Classics and is the symbol of China's highest wisdom. Its core thought is "one Yin and one Yang is called Tao ". Tao constitutes the highest state in ancient philosophy. "Tao" what? The word "Tao" here refers to regular, correct laws. It means that the rules of yin and yang movement are laws, including the changing laws of all things in the universe, including the life, old age, illness and death. If you master the law of change of yin and yang, you can naturally grasp the law of change of everything in the universe. After you mastering the law, then everything can be cured, there will be no difficult problem cannot be overcome. In modern physical education, the dialectical law of yin and yang has been playing a silent role. However, due to the lack of success in the practice of education and control of the laws of Yin and Yang operation and movement, there are many examples of failure in education. Modern education pays attention to 
scores, which are the only basis for success and the only purpose of education in China. The goal of pragmatism pursuing high scores is itself nothing wrong, but the key to the problem is that in the process of score-oriented education, the common phenomenon from family to school to society is the confusion of tools, means and ends. If the score is the "yang" of the leading education, the carrier of the score - the person who is undoubtedly "yin ", is the existence of the opposite of" yang". The source of the score is from time to space, from the classroom to the classroom, from the school to the family all point the arrow to the carrier of this score "person ", which makes the heart bear too much hope, sustenance and role expectation, the body and mind appear serious disharmony. "One yin and one yang is Tao ", which emphasizes the unity of opposites of yin and yang, but in the process of school education, over-emphasizing the importance of achievement and neglecting the health of body and mind is equal to overmagnifying the purpose of score and eliminating the value of human being. This appears in the philosophical level of the "solitary Yin does not live, only Yang does not grow" situation, that is, only pays attention to represent the "Yang" score, sacrifice on behalf of the "Yin" people's physical and mental health practice, is to violate the law. Violation of the law will be punished by the law, so in recent years, there are more and more unsound personality, physical and mental disunity of the problem of college students.

The transformation of human nature by sports is not only the unity of morphological structure and physiological function, but also the unity of body and heart. Physical education should pay attention to the invisible psychological development of students while pursuing their physical transformation. Therefore, physical education should be good at creating a lively teaching atmosphere different from intellectual education teaching, and provide a good environment for students' mental health development. We should be good at making use of the attraction contained in sports activities, and make this attraction multiply and enlarge through reasonable teaching organization. Physical education should be a kind of happy teaching, emphasizing the active participation of the process, emphasizing the positive experience of emotion, emphasizing the independent liberation of personality, so that the interpersonal relationship can be relaxed and harmonious, so that the students can get the healthy development of body and mind freely, carefree and unconsciously in the relaxed and bright environment, in the happy and pleasant mood. Physical education is conducive to promoting the health and perfection of personality. The purpose of physical education is that physical education itself has the function of promoting physical and mental health, and physical and mental health is expressed through the harmony of physical and mental. Physical and mental harmony specifically refers to body and body, meaning and harmony, people and three aspects. "Body and mind" refers to the relative balance of physical and mental health, so that the body and mind to maintain consistency, in order to ensure the overall health of people. And "meaning and" refers to the positive, optimistic, sunshine and other health characteristics in the psychological level, this level of health can only promote and supplement the balance of psychology only when the body's physiology reaches health, so "meaning and" and "body and" are mutually reinforcing and cooperating. These two aspects complement each other, and then make individuals with healthy body and mind enter the social circle of interpersonal communication with friendly, optimistic and positive mental outlook, and promote the good interaction and communication of interpersonal. Social capital is the most important part of productive forces and the core competitiveness in the process of social competition. In the era of paying attention to social capital, a social individual with physical and mental health will obtain more abundant resources in the social resource network, which plays an extremely important role in the development of the individual and the progress of the society.

\section{B. The latent function of physical education to college students' physical and mental development}

The latent function of physical education to college students' physical and mental development is to reserve sustainable development talents for the construction of the motherland. In the competitive, learning pressure of the university campus, there are many students because of academic, employment, economic problems, interpersonal relations and other aspects of the pressure, it is easy to make the original psychological quality of college students cannot correctly face many difficulties, timely relief of psychological pressure, relieve bad mood, and produce bad ideas. Psychologists believe that sports have a "safety valve" role. Through sports, such as through ball games, running, mountain climbing, competition and other activities will relieve bad mood, release psychological pressure. At the same time, to participate in sports, not only can, adjust qi and blood, dredge meridians, effectively exercise the body, but also through sports, can cultivate students' will, quality, in the activities to promote unity, cooperation ability, but also through the way of transposition thinking to see the problem. If you do not participate in sports, cannot release, eliminate, it is easy to backlog in the psychological, once the backlog of negative emotions too much, burst out, the consequences are unthinkable. Only when the body and mind of college students are healthy at the same time, so that the growth of the body and mind into a virtuous circle, can we achieve the "one Yin and one Yang is the way" of talent health view. Only in this way can we reserve and 
transport sustainable development talents for the construction of the motherland.

The latent function of physical education on the physical and mental development of college students adds healthy human capital to the society. Human capital is the capital embodied in the laborer, such as the knowledge and skills of the laborer, the level of culture and technology and the health status, etc. The export terminal of education is to build healthy human capital. The construction of human capital, only in the guarantee of healthy physique, sound personality and good mind, the basic knowledge, basic skills are effective, but also useful. Otherwise, if there is no health system to do security, always appears as sick body, its knowledge and skills are also not normal play, in the health index requirements, not only refer to the health of the body, but also require mental health. Only if all of these are up to standard, can knowledge and skills be used efficiently and become long-term and sustainable human capital.

\section{CONCLUSION}

Under modern education, there are many phenomena that only emphasize scores and achievements, despise students' body, which leads to the imbalance of yin and yang in talent education and talent training. In order to put an end to this phenomenon, we must correct this extreme act as soon as possible. In the context of "great health ", we should take health as the purpose, take physical exercise as the grasp, and promote the all-round development of students. Physical education is the most approachable and operable interventional discipline, which can make a professional responsibility to alleviate and prevent this serious phenomenon. The multiple functions of sports will be paid more and more attention.

\section{References}

[1] Xu Yong, A Common Mental Health Problem for Children and Adolescents, Chinese School Hygiene, 2006 No.5.

[2] Wu Guanghong, Analysis and Countermeasures on the Mental Health of Chinese College Students, Journal of Shanxi Normal University Institute of Physical Education, No.04,2002.

[3] Hou Junsheng, A course in Western Sociology Theory, Nankai University Press, 2010, p.175.

[4] Yang Qingzhong. A study of the classics of Zhou Yi [M]. Beijing: Commercial Press, 2005:199[2]

[5] Introduction to Sports Writing Group. Introduction to Sports [M]. Beijing: Beijing University of physical Education Press, 2013:12.

[6] Hu Jin. The Important Role of Physical Education in Promoting the All-round Development of Students, 2015, (17)P:59 - 60. 